Disponível em:

http://editora.unoesc.edu.br/index.php/race

Race, Joaçaba, v. 15, n. 3, p. 821-842, set./dez. 2016

\title{
A RELAÇÃO DA PREOCUPAÇÃO AMBIENTAL COM O CONSUMO DE PRODUTOS VERDES NO VAREJO
}

The relationship of environmental concern with green products consumer in retail

Sergio Silva Braga Junior

E-mail: sergio@tupa.unesp.br

Doutor em Administração pela Universidade Nove de Julho; Mestre em Administração de Organizações pela Universidade de São Paulo; Professor do Curso de Administração e do Programa de Pós-Graduação em Agronegócio e Desenvolvimento da

Universidade Estadual Paulista.

Endereço para contato: Rua Quirino de Andrade, 215

01049-010, São Paulo, São Paulo, Brasil.

Edgard Monforte Merlo

E-mail: edgardmm@usp.br

Doutor em Administração pela Universidade de São Paulo; Mestre em Economia e Administração pela Universidade de São Paulo.

Dirceu da Silva

E-mail: dirceuds@gmail.com

Doutor em Educação pela Universidade de São Paulo; Mestre em Física pela Universidade de São Paulo.

Artigo recebido em 14 de novembro de 2015. Aceito em 13 de junho de 2016. 


\section{Resumo}

O objetivo com a presente pesquisa foi avaliar a relação da preocupação ambiental do consumidor com sua efetiva declaração de compra de produtos verdes no varejo. Orientado por uma linha de pesquisa que busca explicar o fato de o comportamento ter como precedente a intenção e não a atitude, foi avaliado se a preocupação dos consumidores com o meio ambiente está sendo convertidas em intenção de compra e posterior declaração de compra. O problema que orientou a pesquisa pode ser expresso pela seguinte questão: a preocupação ambiental está relacionada com a intenção de compra para se tornar compra declarada de produtos verdes no varejo? Para responder a essa questão e atender ao objetivo proposto, foi realizada uma pesquisa de natureza quantitativa por meio de um survey com 1.233 consumidores reais de quatro capitais brasileiras, avaliando-se a percepção que eles possuem de si mesmos e dos outros. A análise de dados foi feita utilizando-se a modelagem de equações estruturais por meio do software SmartPLS 3.0. Os resultados indicam que os consumidores pesquisados não demonstram relacionar diretamente sua preocupação ambiental com a compra declarada de produtos verdes, que revelou ser formada pela intenção de compra. Isso foi observado nas duas condições, quando os consumidores responderam sobre a sua percepção e sobre a dos outros (sociedade).

Palavras-chave: Consumo verde. Preocupação ambiental. Varejo. Comportamento do consumidor.

\section{Abstract}

The purpose with this research was to evaluate the environmental consumer concern with their actual purchase statement for green products in retail. Guided by a line of research that seeks to explain the fact that the behavior has as a precedent the intention and not the attitude was assessed to consumer concern about the environment and these are converted into purchase intent and purchase later statement. The problem oriented research can be expressed by the following question: Environmental concern is related to the purchase intention to become declared purchase of green products at retail? To resolve this matter and meet the proposed objective, a quantitative research through a survey with the 1233 actual consumers of four brazilian capitals assessing his perception as an individual was carried out and as he watches others. Data analysis was performed using the structural equation modeling through SmartPLS 3.0 software. The results indicate that consumers surveyed do not demonstrate directly relate their environmental concerns with the purchase declared for green products, which were shown to be formed by purchase intent. This perception takes place in the two conditions, when they respond on their perception and when they answer about how he observes others (society). Keywords: Green consumption. Environmental concern. Retail. Consumer behavior. 


\section{INTRODUÇÃO}

Os conceitos de marketing verde e de consumidor verde não são novos, pois remontam à década de 1980 (CHERIAN; JACOB, 2012). Mas é notório que apenas ao longo da última década se tem aumentado a adoção de comportamentos verdes pelos consumidores, a partir da inserção de produtos e práticas que respeitam mais o meio ambiente (ZAHARIA; ZAHARIA, 2014). Essas questões de interesse ambiental estão ganhando cada vez mais espaço no mundo dos negócios e na academia. Esse movimento ocorre em razão do surgimento de preocupações que atingiram as indústrias, os varejistas e os consumidores sobre o impacto ambiental ocasionado pela sociedade pós-moderna. Para Crumpei, Boncu e Crumpei (2014), valores, crenças e ética pessoal são ingredientes motivadores para determinar as ações das pessoas quanto à preocupação ambiental, além de aumentar o conhecimento sobre o consumo verde. Neste processo, os atores são de fundamental importância para o meio ambiente e a sobrevivência dos negócios (JANSSON; MARELL; NORDLUND, 2010).

Mesmo assim, consumidores têm mostrado que o fato de que mesmo havendo maiores tendências à preocupação ambiental, não necessariamente isso significa que haverá uma clara opção por produtos verdes, como tem sido demonstrado por alguns estudos que associam as preocupações ambientais com a compra de produtos verdes (BRAGA JUNIOR; SILVA, 2013; BRAGA JUNIOR; SILVA, 2014). Essa situação há muitos anos encontrava explicações no trabalho de Bagozzi (1981), em que a relação de comportamento e atitude é indireta e deve ter como mediadora a intenção de compra para se transformar em algo concreto.

Assim, o problema de pesquisa que orienta o presente trabalho pode ser expresso pela seguinte pergunta: as preocupações ambientais influenciam a compra de produtos verdes no varejo? Nesse sentido, o objetivo foi identificar se o consumidor está reconhecendo e efetivamente declarando que compra produtos verdes no varejo.

As hipóteses surgiram a partir da literatura e indicam que o comportamento gera a intenção, e isso gera a atitude dos consumidores para comprar produtos verdes no varejo. O modelo de pesquisa foi montado sob essa estrutura; considerou-se a preocupação ambiental como comportamento, e a intenção de compra e a compra declarada como atitudes. Dado o objetivo, um estudo exploratório de natureza quantitativa foi realizado por intermédio de uma pesquisa com uma amostra de 1.233 respondentes. 


\section{PRODUTOS VERDES}

Com o aumento da participação do varejo na economia e com a crescente preocupação ambiental e sua influência em todos os setores econômicos, incluindo empresas do setor de varejo, é possível observar que nesse setor buscam-se soluções em produtos ecologicamente corretos, ou seja, produtos verdes e orgânicos para atender à demanda de um consumidor preocupado com o meio ambiente e com um estilo de vida mais saudável.

A preocupação em definir os aspectos que diferenciam um produto verde de um produto convencional tem sido objeto de estudo em várias pesquisas (MANUELA, et al., 2013; AR, 2012; BRAGA JUNIOR; SILVA, 2013). Grandes varejistas estão lançando produtos que têm um design distinto, um volume maior ou menor de embalagens, com a finalidade de servir o apelo ecológico e sustentável.

Para se diferenciarem os produtos convencionais dos produtos verdes, é importante saber que estes não são considerados nocivos ao ambiente e à saúde humana, tanto em relação ao seu conteúdo quanto à sua embalagem (JACOBI, 2006).

Assim, um produto verde pode ser considerado aquele que cumpre as mesmas funções do produto convencional equivalente, mas causando menos danos ao meio ambiente durante a sua composição e todo o seu ciclo de vida, considerando-se também a sua embalagem. No entanto, deve-se agregar aos conceitos ecológicos as dimensões políticas, a capacidade de resposta social e o comércio justo.

Pode-se notar que os consumidores de produtos verdes não apenas escolhem os produtos por seu apelo verde, mas pelos benefícios que eles oferecem (MOHR; WEBB, 2005). Embora nenhum produto de consumo possa estar livre de causar impactos prejudiciais ao meio ambiente, a expressão "produto verde” é usada para produtos desenvolvidos para se proteger e/ou melhorar o meio ambiente, à economia de energia e/ou de recursos naturais e à redução ou eliminação de agentes e resíduos tóxicos poluentes. Isso faz com que nos estudos mais próximos das linhas tradicionais sobre o comportamento do consumidor se observe o apelo ético e ambiental desses produtos. Um fator decisivo para a compreensão desse processo é aceitar que as pessoas, muitas vezes, consideram os valores quando elas, de fato, não têm que tomar uma decisão de compra.

Mesmo sem confirmar o reforço dos apelos éticos e ambientais do consumo e da prevalência de aspectos cognitivos na decisão de compra, as empresas estão mais dispostas a apostar no mercado de produtos verdes, focando seus esforços de marketing precisamente nesses aspectos cognitivos. Esse é um ponto que demonstra, 
cada vez mais, o crescimento do consumo verde e o fato de que as empresas estão investindo em produtos que atendam a esses aspectos de mercado.

Assim, diante da revisão apresentada, formulam-se as três primeiras hipóteses desta pesquisa:

H1. A preocupação ambiental do indivíduo se transforma em intenção de compra dele de produtos verdes;

H2. A intenção de compra do indivíduo se transforma em compra declarada dele de produtos verdes;

H3. A preocupação ambiental do indivíduo se transforma em compra declarada dele de produtos verdes.

\section{INTENÇÃO, ATITUDE E DESEJABILIDADE SOCIAL}

Estudos acadêmicos envolvendo intenção, atitude e comportamento são realizados desde a década de 1930, segundo Ajzen e Fishbein (1977). Contudo, o direcionamento dos estudos estava na psicologia, em que se esperava entender a relação entre atitude e comportamento, que até aquele momento se demonstrava sem significância ou com baixa significância estatística.

Nesse sentido, a discussão da relação entre intenção, atitude e comportamento sob o enfoque do marketing, isto é, como o comportamento humano era refletido em suas decisões de compra, ganhou força na década de 1970 com os trabalhos de Engel, Kollat e Blackwell (1968, 1978), Ajzen e Fishbein (1977) e Bagozzi (1981).

Bagozzi (1981) e Ajzen (1985) demonstraram que a intenção e a consequente atitude são fortemente influenciadas por experiências vividas anteriormente pelo consumidor.

Reforçando esse aspecto, Ajzen (2001) explica que a intenção é formada pelas normas subjetivas (conhecimento do certo e errado adquirido na sociedade), pelo padrão de comportamento (rotina) e pelo controle comportamental percebido (como o indivíduo age na sociedade).

Considerando-se que o indivíduo faz o que, geralmente, tem a intenção de fazer, a intenção de compra, segundo Blackwell, Miniard e Engel (2005), é o julgamento subjetivo sobre qual será seu comportamento futuro para a compra de um determinado produto estimulado pelo mercado.

Esse estímulo do mercado busca transformar a intenção de compra em atitude e comportamento de compra. Ou seja, de acordo com Bagozzi (1981), a atitude 
somente influenciará o comportamento por meio de intenções comportamentais; as intenções influenciarão diretamente um comportamento de primeira ordem e apenas indiretamente influenciarão um comportamento de segunda ordem.

Visto que as atitudes são realizadas em relação a algum aspecto do mundo do indivíduo, para representar sua avaliação sobre produtos verdes (AJZEN; FISHBEIN, 1977), no presente estudo, as medidas de atitude foram as ações as quais o indivíduo concorda em tomar conforme seu comportamento de compra no varejo supermercadista.

Essas ações foram consideradas como comportamento de compra do consumidor e, geralmente, acontecem com base em uma intenção previamente formada. Para entender essa relação, Bagozzi (1981) testou um modelo causal que procura explicar a relação entre atitude e intenção de compra como formadoras de comportamento de compra.

Sobre as oportunidades de preferência e escolha, oportunidades de escolha são, muitas vezes, diretamente observáveis, por outro lado, as preferências são determinadas pelas variações de comportamento que surgem em decorrência de acontecimentos, conforme foi observado por Ozguven (2012).

Direta ou indiretamente, o comportamento pode sofrer influência de fatores de primeira e de segunda ordens que o afetam e que, dependendo do tempo e da situação, tornam o indivíduo mais ou menos influenciado, fazendo-o resgatar certas memórias já vivenciadas. Essa afirmação foi reforçada por Bagozzi (1981) e Ajzen (2001), demonstrando que a intenção e o consequente comportamento são fortemente influenciados pelas experiências anteriores (atitudes).

Tai et al. (2012) analisaram a forma como é construída a intenção de compra para a compra de grupos. Jamaluddin, Hanafiah e Zulkifly (2013) e Yildirim e Aydin (2012) observaram o efeito da marca e a decisão de compra pelos efeitos de anúncios de produtos com descontos, demonstrando, assim, a influência da intenção de compra do consumidor.

De acordo com Ajzen (2001), atitudes são formadas por alguns aspectos dentro do Weltanshauung (visão de mundo) dos consumidores, que representam a avaliação do produto em sua causa. Atitudes são as atividades que os consumidores decidem tomar dentro dos seus comportamentos de compra em supermercados de varejo.

Ajzen (2001) explica que há um consenso geral de que uma atitude representa uma avaliação rápida de um objeto psicológico extraído de dimensões anteriormente atribuídas como algo bom ou mau, perigoso ou benéfico, agradável ou desagradável e simpático ou antipático. $\mathrm{O}$ autor, ainda, explica que uma atitude facilita a adaptação 
dos indivíduos ao meio social em que estão inseridos para que eles expressem e defendam o seu comportamento e se adaptem a ele.

No entanto, existe a possibilidade de que as pessoas que participam de surveys ou apresentam respostas às escalas de atitude estejam apresentando um comportamento politicamente correto para a sociedade, e, portanto, também existe a possibilidade de haver resultados errôneos ou incorretos, no sentido de serem muito distintos das percepções reais dos respondentes. Essa tendência de comportamento politicamente correto é chamada desejabilidade social, pois faz com que um indivíduo se mantenha bem diante das normas culturais atuais (SÂRBESCU; COSTEA; RUSU, 2012; RAHIMOF, 2011), mesmo que não compartilhe esses valores.

De acordo com Poínhos et al. (2008), desejabilidade social corresponde à tendência a se transmitir uma imagem culturalmente aceitável e de acordo com as normas sociais, buscando-se a sua aprovação e evitando-se críticas em situações nas quais se é testado.

Indivíduos com alta desejabilidade social são mais propensos a dar respostas que eles consideram mais aceitas pela sociedade, independentemente de serem verdadeiras ou falsas, negando suas associações pessoais com opiniões ou comportamentos socialmente reprovados.

Ribas Junior, Moura e Hutz (2004, p. 84) definem a desejabilidade social como: “[...] a tendência, das pessoas que participam na investigação psicológica, para responder perguntas com base no que é aceito pela sociedade.” Os entrevistados estão inclinados a dar respostas que eles acham socialmente mais aceitáveis e corretas, mesmo que tais respostas não correspondam às suas atitudes, ou melhor, as suas verdadeiras inclinações permanecem secretas se elas não são socialmente aceitáveis. A desejabilidade social também pode consistir em respostas com a finalidade de agradar o entrevistador (RAHIMOF, 2011).

Na teoria da desejabilidade social se explica que as normas e padrões culturais politicamente corretos afetam as respostas dos sujeitos, especialmente em pesquisa que fomenta o autorrelato, como personalidade, ou pesquisa de conteúdo psicológico (SÂRBESCU; COSTEA; RUSU, 2012).

A influência na resposta ocorre quando normas sociais e culturais afetam as respostas dos sujeitos. Ela pode ser definida como uma perspectiva de tendência para dar respostas positivas quando questionado sobre situações onde a resposta tenderia à ser negativa de acordo com Poínhos et al. (2008).

As distorções causadas pela desejabilidade social também podem estar relacionadas a outras variáveis e características subjetivas, como o humor e a autoper- 
cepção. O autoengano pode ocorrer dentro de uma pesquisa. De acordo com Ribas Junior, Moura e Hutz (2004), isso ocorre quando a influência da desejabilidade social é involuntária, ou seja, o respondente não tem conhecimento de uma resposta parcial. Uma variação da desejabilidade social é a gestão de imagem que ocorre quando o entrevistado manipula a resposta de propósito, e, assim, a imagem pessoal transmitida é controlada.

A exigência de conhecer e avaliar a desejabilidade social é necessária porque esse aspecto pode comprometer a validade e a confiabilidade da pesquisa psicológica e comportamental (RIBAS JUNIOR; MOURA; HUTZ, 2004). Consequentemente, várias escalas de medição da desejabilidade social foram recentemente preparadas.

Assim, buscou-se avaliar o comportamento do consumidor em duas formas de responder às afirmações da escala: como o indivíduo responde sobre a sua atitude e como ele observa a atitude das pessoas em sociedade, com o objetivo de avaliar se as respostas mostraram desejabilidade social.

Nesse sentido, para se minimizar a componente de desejabilidade social, utilizam-se técnicas projetivas. Tais técnicas são formadas por esquemas que fazem com que os respondentes não percebam que estão sendo avaliados (BERGERET et al., 2006). A projeção é um recurso da mente humana de colocar no outro sentimentos, desejos e ideias suas, e, ao exercer tal prática, a técnica consegue lidar de maneira mais fácil com esses aspectos, pois é mais fácil projetar aspectos entendidos como falhas do outro indivíduo (BERGERET et al., 2006). Quando se responde algo, as características da sua personalidade ficam conservadas nas respostas (ANZIEU, 1989), e as avaliações feitas no sentido do outro, na realidade, são as do próprio indivíduo.

Mais recentemente, Braga Junior et al. (2015), seguindo o mesmo princípio de Poínhos et al. (2008), realizaram uma pesquisa sobre o reconhecimento e a recompensa para empresas socioambientalmente responsáveis, na qual os respondentes apresentavam a percepção de como as pessoas na sociedade se comportam quando se trata do referido assunto. Nesse caso, as respostas dos participantes da pesquisa sobre a percepção de como a sociedade se comporta quebraram o viés do comportamento socialmente desejável, e observaram-se diferenças entre as respostas relativas à percepção individual e à percepção sobre o comportamento da sociedade. Em tal estudo, observou-se que, no momento em que o indivíduo passou a avaliar os outros, sentiu-se mais confortável em expressar a opinião pessoal sem se sentir socialmente rejeitado.

Dessa forma, agregando a desejabilidade social aos conceitos da revisão realizada, formulam-se outras três hipóteses desta pesquisa: 
H4. A preocupação ambiental do indivíduo faz com que perceba a intenção de compra de produtos verdes do outro;

H5. A intenção de compra de produtos verdes atribuída aos outros se transforma em compra declarada desses produtos;

H6. A preocupação ambiental do indivíduo faz com que ele perceba a compra declarada de produtos verdes atribuída a outros.

\section{PROCEDIMENTOS METODOLÓGICOS}

Para se alcançar o objetivo proposto neste trabalho, foi realizada uma pesquisa exploratória, de natureza quantitativa, por meio de um survey com uma amostra de 1.233 respondentes das Cidades de São Paulo, Rio de Janeiro, Curitiba e Florianópolis.

Quanto à justificativa do método utilizado, a pesquisa exploratória, com uma amostragem não probabilística, por conveniência e de natureza quantitativa, é caracterizada por uma abordagem em campo buscando as características situacionais apresentadas pelos entrevistados para gerar medidas quantitativas dos atributos observados por estes (AAKER; KUMAR; DAY, 2004). Para a coleta de dados, foi contratada uma empresa especializada em pesquisa de mercado, e, assim, foram coletados os dados de consumidores reais residentes nas quatro cidades anteriormente mencionadas.

Para o modelo proposto que se buscou testar (na Figura 1 apresenta-se a análise dos resultados), foi utilizada a escala de Braga Junior e Silva (2013), que já foi validada e testada em diversas pesquisas e se mostrou confiável para ser utilizada.

Na pesquisa avaliaram-se as intenções de compra dos consumidores por produtos verdes no varejo, comparando-se os resultados obtidos em sua autoavaliação e na avaliação das outras pessoas.

Para a aplicação das questões foram utilizadas escalas tipo Likert com cinco pontos de concordância e discordância, em que 1 significa total discordância e 5 significa acordo total. 
Quadro 1 - Escala utilizada na pesquisa

\begin{tabular}{|c|c|c|}
\hline Constructo & Item & Questão \\
\hline \multirow{16}{*}{ 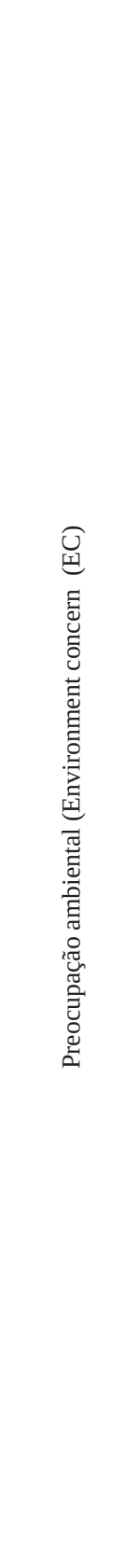 } & EC_1 & $\begin{array}{l}\text { Empresas que prejudicam ou desrespeitam o meio ambiente deveriam ser } \\
\text { punidas. }\end{array}$ \\
\hline & EC_2 & $\begin{array}{l}\text { Defensivos agrícolas e agrotóxicos em alimentos são prejudiciais ao meio } \\
\text { ambiente. }\end{array}$ \\
\hline & EC_3 & Entendo que produtos orgânicos não causam impacto no meio ambiente. \\
\hline & EC_4 & $\begin{array}{l}\text { As certificações ambientais indicam que o fabricante pode apresentar uma } \\
\text { preocupação com o meio ambiente. }\end{array}$ \\
\hline & EC_5 & Estou preocupado com a poluição em minha cidade. \\
\hline & EC_6 & Fico incomodado quando vejo as pessoas sujando os parques e as ruas. \\
\hline & EC_7 & Separo o lixo reciclável do lixo orgânico em minha casa. \\
\hline & EC_8 & O desmatamento pode colocar em risco o futuro da humanidade. \\
\hline & EC_9 & Procuro utilizar transporte público ou andar de bicicleta. \\
\hline & EC_10 & $\begin{array}{l}\text { Sinto que posso ajudar a resolver o problema dos recursos naturais econo- } \\
\text { mizando água e energia. }\end{array}$ \\
\hline & EC_11 & $\begin{array}{l}\text { Sinto que posso proteger o meio ambiente comprando produtos que são } \\
\text { ambientalmente corretos. }\end{array}$ \\
\hline & EC_12 & A emissão de gás carbônico é prejudicial à atmosfera. \\
\hline & EC_13 & Embalagens plásticas e de papelão consomem muitos recursos naturais. \\
\hline & EC_14 & $\begin{array}{l}\text { Embalagens plásticas e de papelão devem ser recicladas e não descartadas } \\
\text { no meio ambiente. }\end{array}$ \\
\hline & EC_15 & $\begin{array}{l}\text { Produtos químicos domésticos (detergentes e produtos de limpeza) são } \\
\text { prejudiciais ao meio ambiente após seu uso. }\end{array}$ \\
\hline & EC_16 & Procuro reutilizar as embalagens dos produtos sempre que possível. \\
\hline
\end{tabular}




\begin{tabular}{|c|c|c|}
\hline \multirow{15}{*}{ 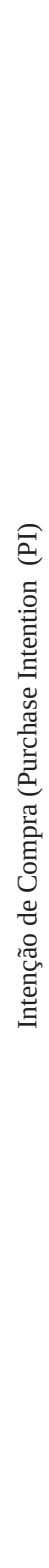 } & PI_1 & Quando possível, procuro escolher produtos que causam menor poluição. \\
\hline & PI_2 & $\begin{array}{l}\text { Estou disposto a evitar produtos fabricados que prejudicam ou desrespei- } \\
\text { tam o meio ambiente. }\end{array}$ \\
\hline & PI_3 & $\begin{array}{l}\text { Estou disposto a comprar alimentos sem agrotóxicos porque eles respeitam } \\
\text { o meio ambiente. }\end{array}$ \\
\hline & PI_4 & $\begin{array}{l}\text { Estou disposto a pagar um pouco mais por produtos e alimentos que estão } \\
\text { livres de elementos químicos e que prejudicam o meio ambiente. }\end{array}$ \\
\hline & PI_5 & $\begin{array}{l}\text { Minha intenção de compra para produtos ecologicamente corretos, avalia a } \\
\text { diferença no preço. }\end{array}$ \\
\hline & PI_6 & $\begin{array}{l}\text { Estou disposto a pagar mais para comprar produtos orgânicos pois não } \\
\text { causam impacto no meio ambiente. }\end{array}$ \\
\hline & PI_7 & $\begin{array}{l}\text { Estou disposto a dar preferência a produtos com informações sobre as } \\
\text { certificações ambientais dos fabricantes. }\end{array}$ \\
\hline & PI_8 & $\begin{array}{l}\text { Procuro considerar se o produto que pretendo comprar não prejudica o } \\
\text { meio ambiente ou outras pessoas. }\end{array}$ \\
\hline & PI_9 & Estou disposto a comprar produtos concentrados. \\
\hline & PI_10 & $\begin{array}{l}\text { Estou disposto a comprar produtos compactados para reduzir a emissão de } \\
\text { gases na atmosfera. }\end{array}$ \\
\hline & PI_11 & $\begin{array}{l}\text { Estou disposto a comprar produtos com pouca embalagem para reduzir o } \\
\text { consumo de recursos naturais. }\end{array}$ \\
\hline & PI_12 & $\begin{array}{l}\text { Estou disposto a evitar a compra de produtos com embalagens que não são } \\
\text { biodegradáveis. }\end{array}$ \\
\hline & PI_13 & $\begin{array}{l}\text { Estou disposto a comprar produtos químicos domésticos (detergentes e pro- } \\
\text { dutos de limpeza) que sejam ecologicamente corretos ou biodegradáveis. }\end{array}$ \\
\hline & PI_14 & $\begin{array}{l}\text { Estou disposto a comprar produtos em “refil” para aproveitar a embalagem } \\
\text { anterior. }\end{array}$ \\
\hline & PI_15 & $\begin{array}{l}\text { Estaria disposto a comprar alguns produtos (agora comprados em tamanhos } \\
\text { menores) em pacotes maiores com menor frequência. }\end{array}$ \\
\hline
\end{tabular}




\begin{tabular}{|c|c|c|}
\hline \multirow{14}{*}{ 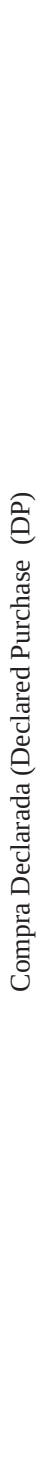 } & DP_1 & $\begin{array}{l}\text { Sempre que compro um produto, verifico se é de empresas que prejudicam } \\
\text { ou desrespeitam o meio ambiente. }\end{array}$ \\
\hline & DP_2 & $\begin{array}{l}\text { Sempre compro alimentos sem agrotóxicos pois sei que estou contribuindo } \\
\text { para o meio ambiente. }\end{array}$ \\
\hline & DP_3 & Pago mais para comprar produtos que promovam a proteção ambiental. \\
\hline & DP_4 & Eu compro produtos orgânicos porque são mais saudáveis. \\
\hline & DP_5 & Pago mais para comprar produtos orgânicos pois são mais saudáveis. \\
\hline & DP_6 & $\begin{array}{l}\text { Compro produtos com certificação ambiental porque são ambientalmente } \\
\text { corretos. }\end{array}$ \\
\hline & DP_7 & $\begin{array}{l}\text { Sempre escolho o produto que é menos prejudicial às outras pessoas e ao } \\
\text { meio ambiente, quando avalio dois produtos concorrentes. }\end{array}$ \\
\hline & DP_8 & $\begin{array}{l}\text { Sempre compro produtos concentrados pois podem economizar àgua e } \\
\text { energia. }\end{array}$ \\
\hline & DP_9 & $\begin{array}{l}\text { Compro produtos compactados para contribuir com a redução da emissão } \\
\text { de gases e por serem mais fáceis para transportar. }\end{array}$ \\
\hline & DP_10 & Sempre compro produtos com menos embalagem possível. \\
\hline & DP_11 & $\begin{array}{l}\text { Compro produtos químicos domésticos (detergentes e produtos de limpeza) } \\
\text { que sejam ecologicamente corretos ou biodegradáveis. }\end{array}$ \\
\hline & DP_12 & Compro produtos em “refil” para aproveitar a embalagem anterior. \\
\hline & DP_13 & $\begin{array}{l}\text { Sempre compro produtos com um design de embalagem não tradicional, } \\
\text { pois pode gerar menos resíduos sólidos. }\end{array}$ \\
\hline & DP_14 & Na compra, eu já troquei ou deixei de usar produtos por razões ecológicas. \\
\hline
\end{tabular}

Fonte: Braga Junior e Silva (2013).

Para a apresentação aos respondentes, foram colocadas duas colunas à direita das assertivas: Você e Outros. Para a coluna Você, os participantes foram convidados a atribuir notas de 1 a 5 para as assertivas, observando se concordavam ou discordavam. Para a coluna Outros, os participantes foram convidados a atribuir notas de 1 a 5, avaliando com quais assertivas as outras pessoas (sociedade) poderiam discordar 
ou concordar. Por meio dessa segunda, coluna tentou-se avaliar o comportamento de desejabilidade social dos respondentes.

O software SPSS 22.0 foi utilizado para a análise de frequências, e o SmatPLS 3.0 - M3 foi utilizado para avaliar a modelagem de equações estruturais (Structural Equation Modeling (SEM), pois os dados eram provenientes de uma escala Likert e atendiam à condição de normalidade multivariada (não aderente) que os modelos de equações estruturais baseados em covariância exigem (RINGLE; WENDE; WILL, 2014). Na verdade, o modelo avalia as relações causais entre as construções e o teste de hipóteses subsequentes avaliando coeficientes entre os constructos.

Para a avaliação do tamanho da amostra mínima e adequada para a análise do modelo apresentado (na Figura 1 apresenta-se a análise dos resultados), seguiram-se as recomendações de Ringle, Silva e Bido (2014), em que o constructo que "recebe mais setas" (preditores) é aquele que define o tamanho em questão. Analisando-se a Figura 1, constata-se que o constructo com mais preditores é o Compra Declarada (DP), com duas setas chegando a ele. Assim, foi utilizado o software G*Power 3.1.7; com as especificações de Cohen (1988) para a área de Ciências Sociais e do Comportamento, isto é, Tamanho do Efeito Médio $(0,15)$ e poder do teste de 0,80 , tem-se que seria necessária para uma escala como a utilizada na pesquisa uma amostra mínima de 68 respondentes.

Essa amostra seria suficiente para se detectarem os efeitos desejados da Modelagem de Equações Estruturais com o Método de Mínimos Quadrados Parciais (Partial Least Square (PLS). Como a amostra desta pesquisa foi de 1.233 respondentes, ficou 18,13 vezes o tamanho calculado. Como já comentado, a referida amostra foi composta por consumidores reais, e os dados foram e foi coletadados por uma empresa especializada em pesquisa de opinião e de marketing.

Assim, empregou-se o Modelo de Medidas para a SEM por mínimos quadrados parciais (Partial Least Square (PM) pela complexidade do modelo a ser testado (HAIR JUNIOR et al., 2014). Também foi realizado o teste PK de Mardia de aderência dos dados que, a uma distribuição normal multivariada, mostrou-se significante ( $\mathrm{p}<0,001)$, indicando que os dados não têm aderência à normalidade multivariada e devem ser tratados com métodos e técnicas que não exijam tal pressuposto.

Há outros modelos de medidas para o cálculo da SEM salvo aqueles com distribuição assintótica livre, ou melhor, sem necessidade de normalidade multivariada. Três modelos podem ser usados: Mínimos Quadrados Ponderados Diagonalizados (DWLS), Mínimos Quadrados Ponderados (WLS) e PLS (HAIR JUNIOR et al., 2013). Os dois primeiros exigem amostras extensas, ou seja, no mínimo, o dobro do 
obtido na pesquisa atual. Eles foram eliminados. PLS - PM foi uma possibilidade adequada para a análise de dados, pois é uma alternativa de qualidade comprovada pela comunidade internacional de pesquisa em diversas áreas do conhecimento (RINGLE; WENDE; WILL, 2014), com grande flexibilidade na análise de dados.

\section{ANÁLISE DOS RESULTADOS}

A análise dos questionários válidos revelou que 52,67\% dos participantes da amostra são mulheres e 47,32\%, homens; a idade média é de 32,9 anos; DP = 10,05 anos; 72,5\% são casados ou têm união estável; 64,8\% frequentam supermercados pelo menos duas vezes por semana; 62,1\% apresentam-se na faixa de renda de cinco a sete salários mínimos mensais. Na proporção de entrevistados por cidade, obteve-se: São Paulo, 26,6\%, Rio de Janeiro, 24,33\%, Florianópolis, 24,74\% e Curitiba, (24,33\%). Os respondentes podem ser entendidos como consumidores reais.

Como foi discutido, para a análise dos dados foi utilizado o software SmartPLS 3.0. Partindo do modelo criado a partir da geração de hipóteses, o modelo foi testado e corrigido por meio da remoção de itens que não apresentavam cargas fatoriais acima de 0,50 (HAIR JUNIOR, 2014). Essa referência é necessária para atender à validade convergente estipulada pelo critério de Fornell e Larcker em que a Variância Média Extraída (Average Variance Extracted (AVE) deve ser maior que 0,50 (HENSELER; RINGLE; SINKOVICS, 2009).

Em sequência às análises, avaliaram-se os $\mathrm{R}^{2}$ (parte das variáveis que explicam os constructos e indicam a qualidade do modelo de ajustamento. Para a área de ciências sociais e comportamentais, Cohen (1988) sugere que $\mathrm{R}^{2}=2 \%$ seja classificado como efeito pequeno, $\mathrm{R}^{2}=13 \%$ como efeito médio e $\mathrm{R}^{2}=26 \%$ como efeito grande. Também, o alfa de Cronbrach (consistência interna) e Confiabilidade Composta são usados para avaliar se a amostra está livre de vieses, ou se as respostas como um todo são confiáveis.

O tamanho do efeito ou indicador de Cohen $\left(\mathrm{f}^{2}\right)$ avalia como cada construção é “útil” para o ajuste do modelo. Os valores 0,02, 0,15 e 0,35 são considerados pequenos, médios e grandes, respectivamente. A validade preditiva $\left(\mathrm{Q}^{2}\right)$ ou indicador de Stone-Geisse avalia a precisão do modelo de ajuste. Os critérios de avaliação são valores maiores que zero (HAIR JUNIOR et al., 2013). Os referidos indicadores de qualidade estão expressos na Tabela 1. 
Tabela 1 - Critérios de qualidade de ajuste de modelos de especificação SEM, Variância Extraída Média (AVE), Confiabilidade Composta, $\mathrm{R}^{2}$, Alfa de Cronbach, Validade preditiva $\left(\mathrm{Q}^{2}\right)$ ou indicador de Stone-Geisser e Tamanho do efeito ( $\left.\mathrm{f}^{2}\right)$ ou Indicador de Cohen

\begin{tabular}{|c|c|c|c|c|c|c|c|}
\hline & AVE & $\begin{array}{r}\text { Conf } \\
\text { co } \\
\end{array}$ & $\begin{array}{l}\text { dade } \\
\text { ta }\end{array}$ & R2* & $\begin{array}{c}\text { Alfa de } \\
\text { Cronbach }\end{array}$ & Q2 & f2** \\
\hline DP_Ind & 0,503 & & 0,890 & 0,423 & 0,859 & 0,206 & 0,348 \\
\hline DP_Other & 0,517 & & 0,865 & 0,325 & 0,813 & 0,165 & 0,311 \\
\hline EC_Ind & 0,537 & & 0,913 & $* * * *$ & 0,892 & 0,436 & 0,409 \\
\hline IP_Ind & 0,506 & & 0,878 & 0.499 & 0,837 & 0,251 & 0,328 \\
\hline IP_Other & 0,517 & & 0,843 & 0.123 & 0,767 & 0,063 & 0,263 \\
\hline $\begin{array}{l}\text { Valores de } \\
\text { referência }\end{array}$ & $>0,50$ & $>0,70$ & & $\begin{array}{l}\text { vide } \\
\text { nota } 1\end{array}$ & $>0,70$ & $\mathrm{Q} 2>0$ & vide nota 2 \\
\hline
\end{tabular}

Fonte: os autores.

Notas: * Valores referenciais para o $\mathrm{R}^{2}$ para área de ciências sociais e comportamentais: $\mathrm{R}^{2}=2 \%$ seja classificado como pequeno, $\mathrm{R}^{2}=13 \%$ como médio e $\mathrm{R}^{2}=26 \%$ como grande (COHEN, 1998).

** Valores de 0,02, 0,15 e 0,35 são considerados pequenos, médios e grandes, respectivamente (HAIR JUNIOR et al., 2013).

A análise da Tabela 1 indica nitidamente que o modelo pode ser considerado ajustado como um todo, pois após a eliminação de variáveis pouco aderentes a ele, obtiveram-se indicadores que atendem aos requisitos de ajuste do PLS - SEM.

Além da qualidade do ajuste do modelo, deve-se analisar a validade discriminante, pois os constructos devem se relacionar, mas ser independentes (HAIR JUNIOR et al., 2013). Usa-se, para tal, o critério de Fornell e Larcker, compara-se as raízes quadradas dos valores AVE para cada construção com as correlações (Pearson) entre os constructos (ou variáveis latentes). As raízes quadradas das AVE devem ser maiores do que a correlação entre os constructos (Tabela 2).

Tabela 2 - Avaliação da validade discriminante - Comparação das raízes quadradas das AVE (em amarelo na diagonal principal) versus correlação entre constructos

\begin{tabular}{lrrrrr}
\hline & DP_Ind & DP_Other & EC_Ind & IP_Ind & IP_Other \\
\hline DP_Ind & 0,709 & & & & \\
DP_Other & 0,645 & 0,719 & & & \\
EC_Ind & 0,418 & 0,168 & 0,733 & & \\
IP_Ind & 0,630 & 0,351 & 0,666 & 0,712 & \\
IP_Other & 0,445 & 0,566 & 0,351 & 0,562 & 0,719 \\
\hline
\end{tabular}

Fonte: os autores. 
A análise da Tabela 2 mostra que as raízes quadradas das AVE são maiores, em todos os casos, do que as correlações entre os constructos. Esse fato mostra que o modelo tem validade discriminante e pode ser interpretado.

Por fim, com o objetivo de avaliar a qualidade geral do modelo, calculou-se o indicador Goodness -of-Fit (GoF), que é dado pela média geométrica do $\mathrm{R}^{2}$ médio e AVE ponderada média (RINGLE; WENDE; WILL, 2005). O valor calculado foi de 0,392 e indicou que o modelo pode ser considerado bem ajustado, uma vez que valores acima de 0,36 são considerados bons para áreas como ciências sociais e comportamentais (WETZELS; ODEKERKEN-SCHRÖDER; VAN OPPEN, 2009).

Após a constatação de que o modelo teve um ajuste adequado, podem-se analisar as hipóteses criadas a partir da teoria (Figura 1).

Nesse modelo é possível observar que a intenção de compra de produtos verdes é influenciada pela preocupação ambiental do indivíduo. Isso também é confirmado quando se analisa o modelo sobre a sua percepção de como os outros se comportam.

Figura 1 - Modelo ajustado da pesquisa

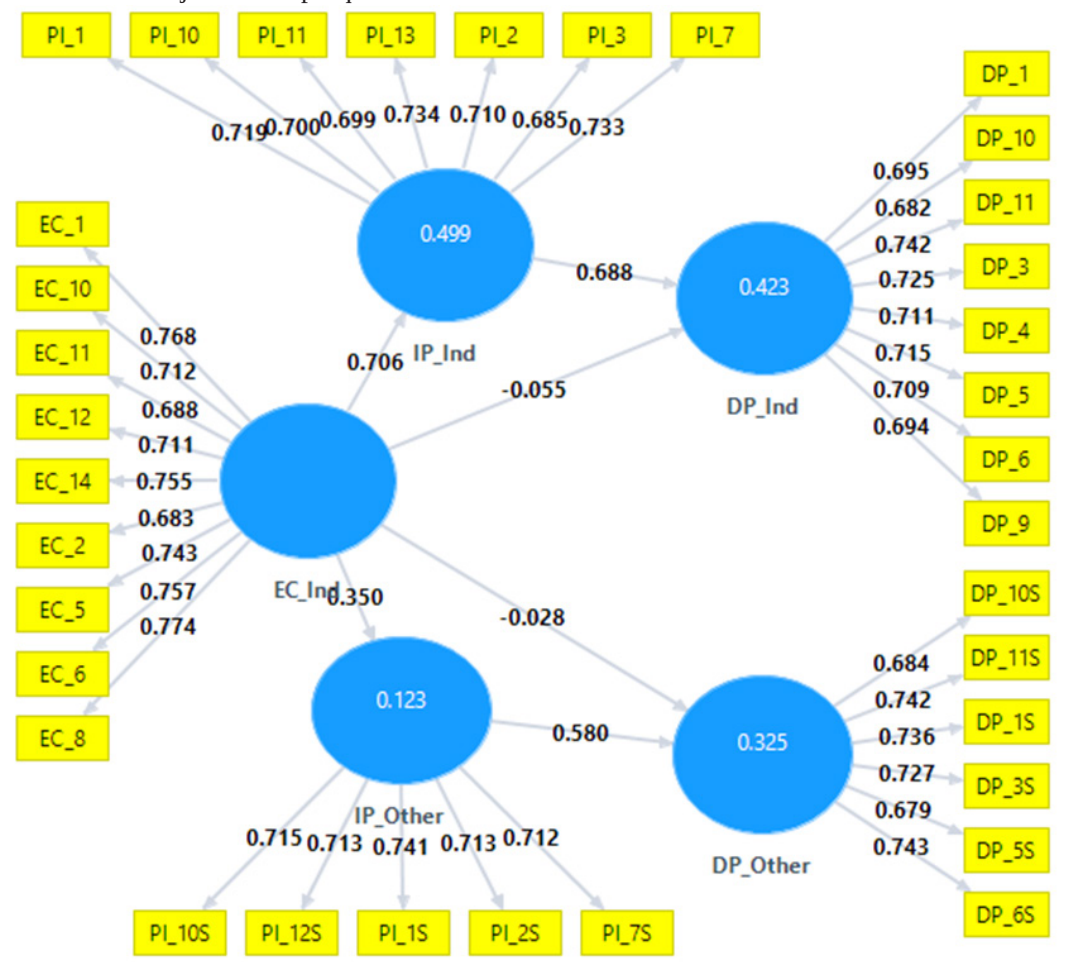

Fonte: os autores. 
A relação entre as questões ambientais e a compra declarada não é confirmada quando o indivíduo é questionado sobre o seu comportamento e quando avalia o dos outros (como a sociedade se comporta). Isso não quer dizer que o comportamento não acontece; demonstra que o comportamento de compra pode acontecer mediante a intenção.

Em uma análise mais detalhada, as variáveis eliminadas do modelo mostraram, em análise descritiva, que possuíam pouca variação, e acabava não ocorrendo uma discriminação passiva de ajuste no modelo. Também foi possível notar que alguns itens foram considerados “verdades absolutas” pelos respondentes.

No caso do constructo “Preocupação Ambiental”, os itens 3, 4, 7, 9, 13 e 15 foram eliminados, pois todos estão relacionados a questões ligadas ao senso comum das pessoas, em que se pode notar que os respondentes não entendem que as ações ligadas a essas questões são de responsabilidade de cada um como indivíduo para formar o todo.

Já em "Intenção de Compra” o único item comum nas respostas para Você e Outros foi o item 12 (PI_12. Estou disposto a evitar a compra de produtos com embalagens que não são biodegradáveis.) que trata sobre embalagens. Quanto aos outros itens, o que ficou ajustado no modelo para Você é o complemento de Outros. É possível perceber que as verdadeiras intenções estão no constructo "PI_Other”, e as ações de intenções estão em "PI_Ind”, ficando evidente o aspecto do "eu faço mas os outros não”.

Essa evidência fica mais perceptível na "Compra Declarada”, em que os itens ajustados no modelo são comuns em “DP_Ind” e “DP_Other”; no “DP_Other” ainda ficam os itens 4 e 9 (DP_4. Eu compro produtos orgânicos porque são mais saudáveis; DP_9. Compro produtos compactados para contribuir com a redução da emissão de gases e por serem mais fáceis para transportar).

Assim, considerando o modelo ajustado, pode-se notar que a prática no consumo de produtos verdes no varejo ainda é limitada, na grande maioria, pelo que está representado no constructo “DP_Other”.

Assim, uma vez confirmados os ajustes e indicadores de qualidade do modelo, a partir dos resultados foi possível fazer inferências sobre os coeficientes de caminho e o p-valor de cada relação causal, indicadas na Figura 1. Como o modelo ajustado, esses valores podem ser usados para avaliar as hipóteses de pesquisa, conforme se apresenta na Tabela 3. 
Tabela 3 - Avaliação das hipóteses

\begin{tabular}{lrrrl}
\hline & Coeficiente de caminho & t-valor & p-valor & Conclusão \\
\hline EC_Ind => DP_Ind & $-0,055$ & 1,593 & 0,112 & Não suportada \\
EC_Ind => DP_Other & $-0,028$ & 0,859 & 0,391 & Não suportada \\
EC_Ind => IP_Ind & 0,706 & 43,324 & 0,000 & Suportada \\
EC_Ind => IP_Other & 0,350 & 12,736 & 0,000 & Suportada \\
IP_Ind => DP_Ind & 0,688 & 21,945 & 0,000 & Suportada \\
IP_Other => DP_Other & 0,580 & 21,612 & 0,000 & Suportada \\
\hline
\end{tabular}

Fonte: os autores.

Nota: A significância foi estimada pelo método de bootstrapping com n =600, 1000 repetições e nível de significância de 5\% (conforme sugerido por Ringle, Wende e Will, 2014).

A H3 foi considerada não suportada por ter um coeficiente de caminho negativo; apesar de ter o p-valor da sua relação causal com o modelo significante em nível de 10\%, a melhor opção pode ser desconsiderá-la. Também, a H6 foi não suportada por apresentar o coeficiente de caminho negativo e ter um p-valor não significante ( $>>0,05)$.

\section{CONSIDERAÇÕES FINAIS}

Muitas vezes, a decisão de compra tomada de forma individual não é o desejado pelo consumidor, mas é a decisão que melhor se adapta à sua necessidade no momento e na sua condição de realidade. Por outro lado, a sociedade que ele idealiza (mitigação da desejabilidade social) não gera condições de adaptação e cobra uma postura que ainda não faz parte da sua realidade.

Avaliar a percepção segundo a intenção de compra do consumidor e a declaração de comprar produtos verdes torna-se relevante, especialmente quando o reflexo da preocupação ambiental na compra declarada não foi verificado.

Assim, considerando-se o propósito da pesquisa, pode-se inferir que a preocupação com o meio ambiente não tem nenhum efeito sobre a compra de produtos verdes declarada. Por outro lado, tem uma relação significativa com a intenção de compra.

Essas inferências podem ser justificadas pela possibilidade de os consumidores não perceberem a importância de mudar o hábito de consumo e manterem a sua rotina de compras que tem experiência do passado, reforçando o fato de que a atitude exerce uma influência baixa sobre o comportamento de compra do indivíduo, como já foi demonstrado por Bagozzi (1981). Nesse sentido, na pesquisa mostrou-se que a preocupação ambiental interfere na intenção de compra e deve ser o foco de estudo para se transformar comportamentos e, consequentemente, atitudes. 
Além disso, os resultados demonstram que as opiniões seguem uma orientação correta, mas sofrem a influência de respostas politicamente corretas e acabam por indicar que as pesquisas na área de gestão ambiental, meio ambiente, sustentabilidade, talvez, devessem ser revistas, porque há uma sensação de que os consumidores estão percebendo o que foi pesquisado.

Assim, os resultados das respostas que o indivíduo afirmou para si e o que ele observa na sociedade deveriam ser quase iguais, porque todos estão na mesma sociedade. Outro aspecto que pode não estar contribuindo para o aumento da oferta de produtos verdes no varejo pode ser conectado ao posicionamento de marketing.

Outro ponto de vista a ser refletido pode ser a possibilidade de os consumidores estarem buscando produtos orgânicos ou ecologicamente corretos em varejos especializados, deixando, assim, de considerar os supermercados como possibilidades de fornecimento de produtos verdes.

Finalmente, a principal contribuição do estudo foi mostrar que o consumidor está comprando os produtos verdes, mas essa tendência pode ser ainda restrita em decorrência do preço elevado e da falta de hábito de consumo desses produtos.

\section{REFERÊNCIAS}

AAKER, D. A.; KUMAR, V,; DAY, G. S. Pesquisa de marketing. São Paulo: Atlas, 2004.

AJZEN, I.; FISHBEIN, M. Attitude-behavior relations: a theoretical analysis and review of empirical research. Psychological Bulletin, v. 84, i. 5, p. 888-918, 1977.

AJZEN, I. From intention to action: a theory of planned behavior. In: KUHL, J.; BECKMAN J. (Ed.). Action control: from cognitions to behaviors. New York: Springer, 1985.

AJZEN, I. Nature and operation of attitudes. Annual Reviews of Psychology, v. 52, p. 27-58, 2001.

ANZIEU, D. Os métodos projetivos. Rio de Janeiro: Campus, 1989.

AR, I. M. The impact of green product innovation on firm performance and competitive capability: the moderating role of managerial environmental concern. Procedia-Social and Behavioral Sciences, v. 62, p. 854-864, 2012. 
BAGOZZI, R. P. Attitudes, intentions, and behavior: a test of some key hypotheses. Journal of Personality and Social Psychology, v. 41, i. 4, p. 607, 1981.

BERGERET, J. et al. Psicopatologia: teoria e clínica. Porto Alegre: Artmed, 2006.

BLACKWELL, R. D.; MINIARD, P. W.; ENGEL, J. F. Comportamento do consumidor. 9. ed. São Paulo: Pioneira Thomson, 2005.

BRAGA JUNIOR, S. S. et al. The effects of environmental concern on purchase of green products in retail. Procedia-Social and Behavioral Sciences, v. 170, p. 99108, 2015.

BRAGA JUNIOR, S. S. et al. Uma análise da consciência ecológica para o consumo "verde” no varejo supermercadista. Revista de Gestão Social e Ambiental, v. 6, n. 2, 2012.

BRAGA JUNIOR, S. S.; SILVA, D. da; AQUINO, N. S. Comportamento do consumidor: há recompensa às empresas socioambientalmente responsáveis? Revista de Administração da UFSM, v. 8, n. 3, p. 384-396, 2015.

BRAGA JUNIOR, S. S.; SILVA, D. da. A relação da preocupação ambiental com compra declarada para produtos verdes no varejo: uma comparação da percepção do indivíduo com sua percepção de sociedade. Perspectivas em Gestão \& Conhecimento, v. 3, n. 2, p. 161-176, 2013.

BRAGA JUNIOR, S. S.; SILVA, D. da. Consumo de produtos verdes no varejo: A intenção de compra versus a compra declarada. Agroalimentaria, v. 20, n. 39, p. 155-170, 2014.

CHERIAN, J.; JACOB, J. Green marketing: a study of consumers' attitude towards environment friendly products. Asían Social Science, v. 8, n. 12, p. 117, 2012.

COHEN, J. Statistical power analysis for the behavioral sciences. $2^{\text {nd }}$ ed. New York: Psychology Press, 1988.

CRUMPEI, I.; BONCU, S.; CRUMPEI, G. Environmental attitudes and ecological moral reasoning in Romanian students. Procedia-Social and Behavioral Sciences, v. 114, p. 461-465, 2014.

ENGEL, J. F.; KOLLAT, D. T.; BLACKWELL, R. D. Consumer behavior. Illinois: Holt, Rinehart \& Winston, 1968. 
HAIR JUNIOR, J. F. et al. A primer on partial least squares structural equation modeling (PLS-SEM). [S.l.]: Sage Publications, 2013.

HENSELER, J.; RINGLE, C. M.; SINKOVICS, R. R. The use of partial least squares path modeling in international marketing. New Challenges to International Marketing Advances in International Marketing, v. 20, p. 277-319, 2009.

JAMALUDDIN, M. R.; HANAFIAH, M. H.; ZULKIFLY, M. I. Customer-based Psychology Branding. Procedia-Social and Behavioral Sciences, v. 105, p. 772780, 2013.

JANSSON, J.; MARELL, A.; NORDLUND, A. Green consumer behavior: determinants of curtailment and eco-innovation adoption. Journal of Consumer Marketing, v. 27, i. 4, p. 358-370, 2010.

MANUELA, V.-Z. et al. The influence of the term 'organic' on organic food purchasing behavior. Procedia-Social and Behavioral Sciences, v. 81, p. 660-671, 2013.

MOHR, L. A.; WEBB, D. J. The effects of corporate social responsibility and price on consumer responses. Journal of Consumer Affairs, v. 39, i. 1, p. 121-147, 2005.

OZGUVEN, N. Organic foods motivations factors for consumers. Procedia-Social and Behavioral Sciences, v. 62, p. 661-665, 2012.

POÍNHOS, R. et al. Desejabilidade social e barreiras ao cumprimento da terapêutica dietética em mulheres com excesso de peso. Acta Med Port, v. 21, p. 221-228, 2008.

RAHIMOF, F. et al. Effects of educational improvements on student social desirability. Procedia-Social and Behavioral Sciences, v. 29, p. 932-935, 2011.

RIBAS JUNIOR, R. de C.; MOURA, M. L. S. de; HUTZ, C. S. Adaptação brasileira da escala de desejabilidade social de Marlowe-Crowne. Avaliação Psicológica, v. 3, n. 2, p. 83-92, 2004.

RINGLE, C. M.; SILVA, D. da; BIDO, D. de S. Modelagem de equações estruturais com utilização do SmartPLS. REMark, v. 13, n. 2, p. 54, 2014.

RINGLE, C. M.; WENDE, S.; WILL, A. SmartPLS 3.0. Germany: University of Hamburg, 2014. 
SÂRBESCU, P.; COSTEA, I.; RUSU, S. Psychometric properties of the marlowe-crowne social desirability scale in a Romanian sample. Procedia-Social and Behavioral Sciences, v. 33, p. 707-711, 2012.

TAI, C.-L. et al. Determinants of consumer's intention to participate in group buying. Procedia-Social and Behavioral Sciences, v. 57, p. 396-403, 2012.

WETZELS, M.; ODEKERKEN-SCHRÖDER, G.; VAN OPPEN, C. Using PLS path modeling for assessing hierarchical construct models: guidelines and empirical illustration. MIS quarterly, p. 177-195, 2009.

YILDIRIM, Y.; AYDIN, O. Investigation of the effects of discount announcements on consumers' purchase decisions: a case study in supermarket. Procedia-Social and Behavioral Sciences, v. 62, p. 1235-1244, 2012.

ZAHARIA, I.; ZAHARIA, C. The greening of consumer culture. Economics, Management, and Financial Markets, i. 1, p. 136-141, 2014.

Agradecimento

Ao Conselho Nacional de Desenvolvimento Científico e Tecnológico (CNPq) pelo apoio financeiro à realização da pesquisa.

Como citar este artigo:

ABNT

BRAGA JUNIOR, Sergio Silva; MERLO, Edgard Monforte. A relação da preocupação ambiental com o consumo de produtos verdes no varejo. RACE, Revista de Administração, Contabilidade e Economia, Joaçaba: Ed. Unoesc, v. 15, n. 3, p. 821-842, set./dez. 2016. Disponível em: <http://editora.unoesc.edu.br/index.php/ race>. Acesso em: dia/mês/ano.

APA

Braga Junior, S. S., \& MERLO, E. M. (2016). A relação da preocupação ambiental com o consumo de produtos verdes no varejo. RACE, Revista de Administração, Contabilidade e Economia, 15(3), 821-842. Recuperado em dia/mês/ano, de http:// editora.unoesc.edu.br/index.php/race 